\title{
BioCore International Journal of Criminal and Forensic
} Exploring Scientific community Science

Copyright: (C2017 John Zheng Wang. This is an open-access article distributed under the terms of the Creative Commons Attribution License, which permits unrestricted use, distribution, and reproduction in any medium, provided the original author and source are credited

Received: September 03, 2017 Accepted: September 14, 2017, Published: September 27, 2017

\section{Abstract:}

Rapid detection and visualization of human saliva stains is one of the challenging tasks in forensic cases, such as murder, rape, sexual assault, and child abuse. A more challenging question is to detect dry saliva stains from certain difficult surfaces, e.g. kitchen paper towels, bitten apples, or bath towels because these item surfaces are considered as absorptive, textured, and fragile materials, rendering saliva either to be evaporated or soaked quickly. The forensic value of such an attempted method lies in the fact that once saliva is detected and visualized, the sampling area can be swapped for a DNA identification. Therefore, a novel examination approach is much needed for both field and lab applications. In this paper, a quasi-experimental study was reported to detect, visualize, and photograph latent dry saliva stains on a piece of kitchen paper towel, a bitten apple, and a piece of bath towel using a new brand of a laser detector $(447 \mathrm{~nm})$. The preliminary results indicated that the laser method could produce a high quality of images of saliva existence almost simultaneously. Further, the portable laser device allows for a non-destructive, non-contact, and non-invasive examination. In sum, this work progresses toward the ultimate goal of rapidly detecting and visualizing human saliva on difficult surfaces in a real-time manner that would otherwise remain undetected using other traditional development methods.

Key Words: Forensic Examination, Latent Biological Evidence, Portable Laser Detector (447 nm), Detection, Visualization, Photo tgraphing, Latent Dry Saliva Stains, Absorptive, Textured, and Fragile Surfaces

\section{Introduction:}

Biological fluid stains recovered at crime scenes are among the most important types of evidence to forensic investigation. A determination of body fluid present is more likely to make the sample to undergo further laboratory testing including DNA analysis to identify a suspect or victim as well as exonerate an innocent individual, which is a very crucial step in a wide range of investigations. Saliva is one of the vital biological fluids secreted in human beings, which can be deposited on a specific surface by specific act, such as biting, sucking, licking, kissing, and possibly through other behaviors ${ }^{[1]}$. As routine tasks at crime scenes and in the labs, quick detection and visualization of saliva stains constitute daily forensic science casework because saliva can be used as potential source of DNA identification. Depending on the type of the surfaces, it is very difficult to detect saliva on certain difficult surfaces, namely, paper tissues, fabric cloth, and/or bite marks on the human skin because these surfaces are absorptive, textured and elastic, making salvia to be soaked or evaporated quickly. Further, dried saliva stains are invisible to the human eye, which adds to difficulty of recognizing and collecting because dried saliva belongs to latent biological evidence ${ }^{[2]}$. While the DNA present in saliva on these surfaces are difficult to collect and extract than similar stains on glass bottles, plastic cups, or door handles, the substrate of these unique objects are commonly encountered at crime scenes. In reality, some of the objects on which saliva is deposited (e.g. human skin) cannot be usually submitted for direct swap and extraction procedures ${ }^{[3]}$. Therefore, a novel and improved detection and visualization method is much needed and demanded to identify invisible dry saliva stains on these surfaces. In turn, other methods of extracting DNA are then flowed to identify the suspect and exclude the innocent ${ }^{[4]}$. To date, various methods for detecting dried saliva stains have been tried out, e.g. chemicals ${ }^{[5]}$, fluorescence spectroscopy ${ }^{[6]}$, lasers ${ }^{[7]}$, but each method has its own strengths and limitations. This paper intends to report the preliminary findings of detecting, visualizing, and photographing dry saliva stains on three difficult surfaces using a new portable laser device with improved technology.

Laser technology, in general, refers to a special device that can emits light through a process via the simulated emission of electromagnetic radiation, or a short term for "light amplification by simulated emission of radiation." Ideally, a better laser device can 
produce a highly concentrated laser beam in three special functions that are different from other optical sources. The space coherence of the laser beam can have little or no divergence, thus focusing on a targeted area. The temporal coherence can emit the light in a single color, most commonly referred to as monochromatic light source, such as blue, green, or red. Finally, the device can be battery-based to scan or sweep a large area at any location. Many types of lasers are being employed for different purposes. One of the improved laser technological methods is the optically pumped semiconductor function based on a diode pump solid-state laser. This new type of laser can produce a more compact, firm, and powerful beam via a portable device with a better and clearer image for photographing. Most importantly, due to the high intensity of the laser beam, the device may detect biological latent residues, e.g. dry saliva in this project on difficult surfaces. Essentially, the narrow laser spectrum can react with dry saliva stains on the targeted surface, producing a fluorescence-excited spectrum of the elemental constituent of the surface material. The super spectral brightness of the laser beam is considered to be much superior to those lamp-based systems, such as the UV lights.

Another feature of the optically pumped semiconductor technology is the fiber-delivery cable that is connected between the device and a laser hand-piece. In other words, to be portable and flexible, the solid-state laser or laser amplifier can render the light to be guided in a single mode optical fiber. In turn, the guided light allows extremely long gain regions, providing good cooling conditions because fibers have high surface area to volume ratio for the efficient cooling. In addition, the fiber's wave guiding properties tend to reduce thermal distortion of the beam. As a result, the extended and flexible cable from the device can be operated from any direction and at any angle. Regarding the construction material, the fiber laser usually has as a double-clad fiber. This type of fiber consists of a fiber core, an inner cladding, and an outer cladding.
The index of the three concentric layers is chosen so that the fiber core acts as a single-mode fiber for the laser emission while the outer cladding acts as a highly multimode core for the pumped laser. Consequently, the pump can emit a large amount of power into and through the active inner core region with a high numerical aperture (NA) for easy launching conditions.

In reality, a fiber disk laser or a stack of such lasers is used to prevent a photo-darkening effect. As a fundamental limit, the intensity of the fiber laser light leads to optical non-linearity (photo-darkening effect), which is induced by the local electric field strength; therefore, it becomes dominant and often causes the material destruction of the fiber. With the new improved technology, small sized laser devices from several companies are being applied in modern forensics.

\section{Materials and the Quasi-Experimental Design:}

The laser detection enjoys several advantages over other technical methods, e.g. Fluorescence spectroscopy (Spectroscopic), RSID-Saliva Test, Amylase ELISA, HTN3, STATH/HTN3 (Immunological), or UV (optical) methods. First, the laser detection is non-destructive by nature (including DNA in saliva) and should be used as the first attempt in detecting latent evidence. Second, the new laser device is portable, and can be used not in the field, but also in the lab. Third, the new technology is a one-step scan application without any complex pre-treatment. Next, the laser detection is a real-time method with a direct and simultaneous viewing result. Finally, the laser device has a potential to detect latent biological saliva stains on many difficult surfaces.

In the current paper, a preliminary investigation is reported under a controlled-setting (quasi-experimental design) using a new brand of laser device. Essentially, the new device (the manufactory information is provided in the acknowledge) is unique in the following features (See Figure 1):

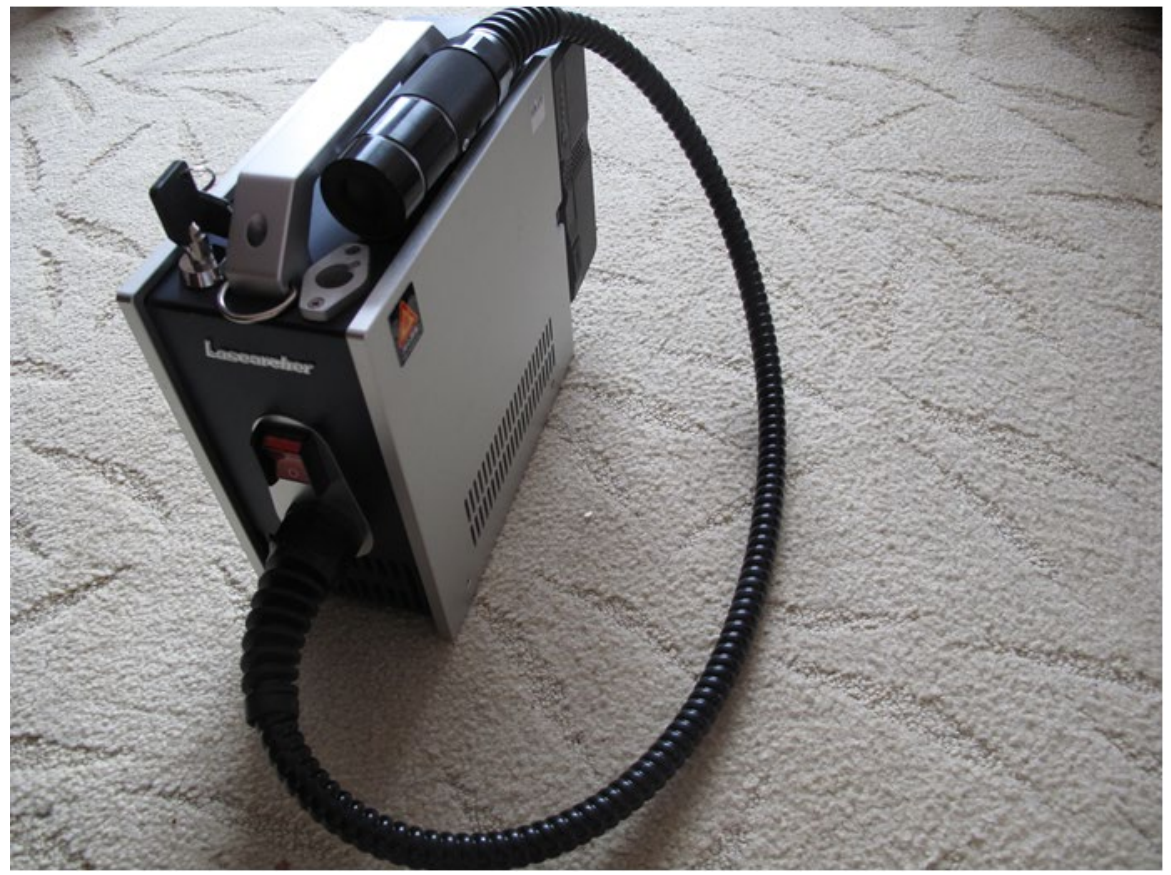

Figure 1: The portable laser device ( $447 \mathrm{~nm} /$ blue or $532 \mathrm{~nm} /$ green) was employed in the quasi-expermental study. 


\section{The Main Component:}

The laser devise has a dual wavelength of $532 \mathrm{~nm}$ and $447 \mathrm{~nm}$ by a switch via diode pump solid-state laser. The device weighs only $3.5 \mathrm{~kg}$ with a dimension of $225 \mathrm{~mm} / 9$ " (length), $105 \mathrm{~mm} / 4.2$ " (width), and $165 \mathrm{~mm} / 6.6$ "(height). Either AC or an inserted DC battery $(1.5 \mathrm{~kg})$ can operate the device. The battery can last $4 \sim 6$ hours for interrupted or continuous operations for an output power of 8- watts and can be rechargeable for 4 hours for another full use. The Delivery System:

The device has a metal covering fiber optical cable $(\mathrm{L}=1.2 \mathrm{~m}$.) or called hand-piece for broad-spectrum. The hand-piece controls the volume of the laser beam and can produce a maximum illumination of a circular area ( $D=50$ centimeters) with no speckles on the target area. The fiber delivery cable renders a $1 \mathrm{~nm}$ color purity bandwidth (green light) that is a huge advantage over the other commercial diode-pumped solid-state laser devices. The device can be used in three different positions depending on the height of potential target areas. If latent evidence is located below the knee, a ground-tripod position can be utilized where the device is placed on the ground and the hand-piece can be placed on the tripod. If latent evidence is located around the chest level, the device should be used by a hand-hand position, meaning that the device is carried by one hand, and the hand-piece is held by the other hand. Finally, if latent evidence is above the eye or in a difficult position, the device can be carried by a backpack position by the stripes attached and the hand-piece is held by the hand to scan a target area.

The Viewing/Photographing Component:

The filter/goggles is required for viewing and photographing, which is made to block certain wavelength due to its OD value $(>7)$ and renders the human eyes to see with a regular digital camera for photographing under the yellow filter/goggles. The device is used in the current project because its laser beam of the required spatial and temporal coherences could be used to detect latent evidence on difficult surfaces, in this case, latent dry saliva stains on three different difficult surfaces. In sum, the device is designed to generate better quality of laser beam with stable wavelength radiation and continuous ultrashort laser energy.

To simulate a real scenario of a crime scene, three types of objects are selected based on a purposive sampling method where prior knowledge or experience could enable the research to select proper samples. A quasi-experimental design is an empirical method estimating the causal impact of a treatment or an intervention on its targeted samples. The design consists of three main components. First, a quasi-experimental research does not use random assignments to treatment or control. Instead, a quasi-experimental design typically allows for the researcher to control the assignments to the treatment condition and use some criterion for practical purposes. Second, the researcher can have control or manipulation over assignments to the treatment due to limitations of time and resources ${ }^{[8]}$. Finally, there are several types of quasi-experimental designs, each with different strengths, weaknesses, and applications ${ }^{[9]}$. Obviously, the quasi-experimental design allows the research to maximize a crime scene scenario with contain level of control for this study. The three pieces of objects were selected because they are commonly seen at crime scenes to contain latent dry saliva stains: a piece of kitchen paper towel, an apple, and a piece of bath towel. Three volunteers were ask to deposit their saliva onto these three objects without telling the author of the exact location(s), which in principle is similar to the so-called black box study.

\section{Quasi-Experiment 1:}

The first volunteer was asked to deposit his saliva by wiping the kitchen paper towel across his mouth back and forth a couple of times to get a good amount of saliva and threw it on the floor. The wiping action was exercised with much force, simulating a scenario where the attacker was trying to use a piece of kitchen paper towel to cover the victim's mouth during a struggle. The sample was on the floor for two hours to let it dry and then was photographed for a comparison purpose. To the naked eye, no saliva stain was visible at this time (See Figure 2).

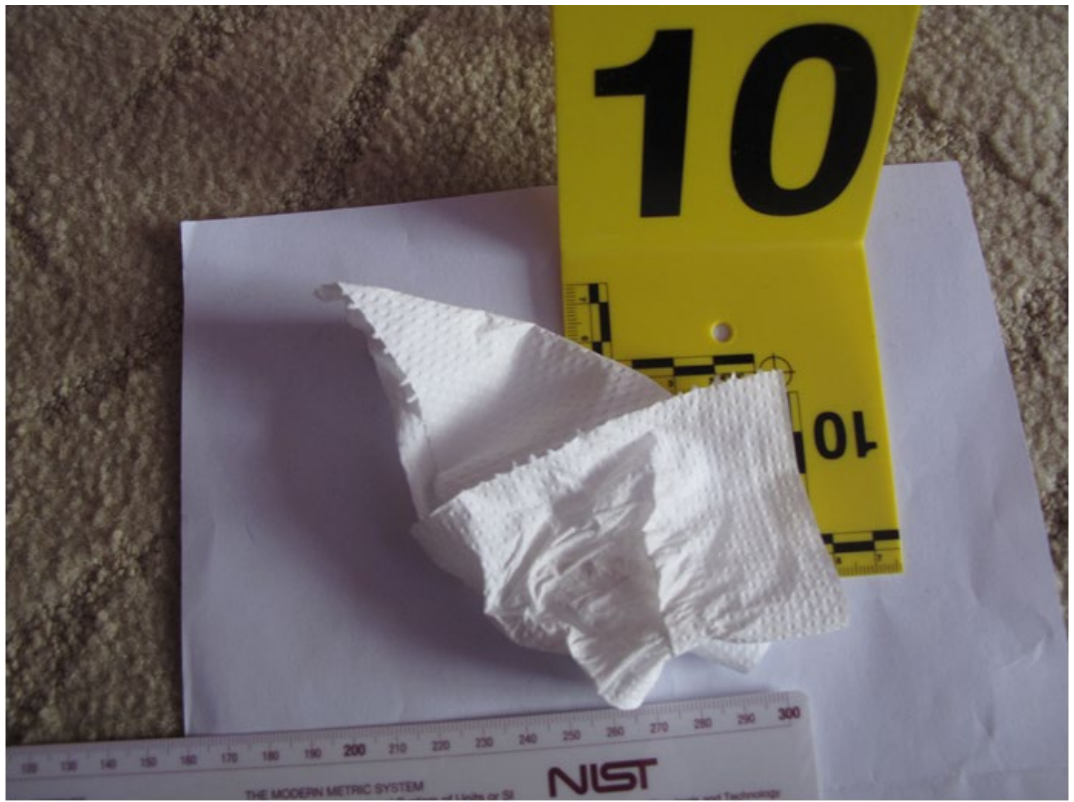

Figure 2: A piece of kitchen paper towel was photographed by a regular digital camera where no saliva stain was visible to the naked eye. 


\section{Quasi-Experiment 2:}

The second volunteer was asked to bite an apple in a free movement without considering too much or too little pressure on the apple. The apple was then thrown onto the floor for two hours and then was photographed for a comparison purpose. The scenario was designed for a common situation where the suspect eats something at a crime scene. To the naked eye, no latent saliva stain was visible at the time (See Figure 4).

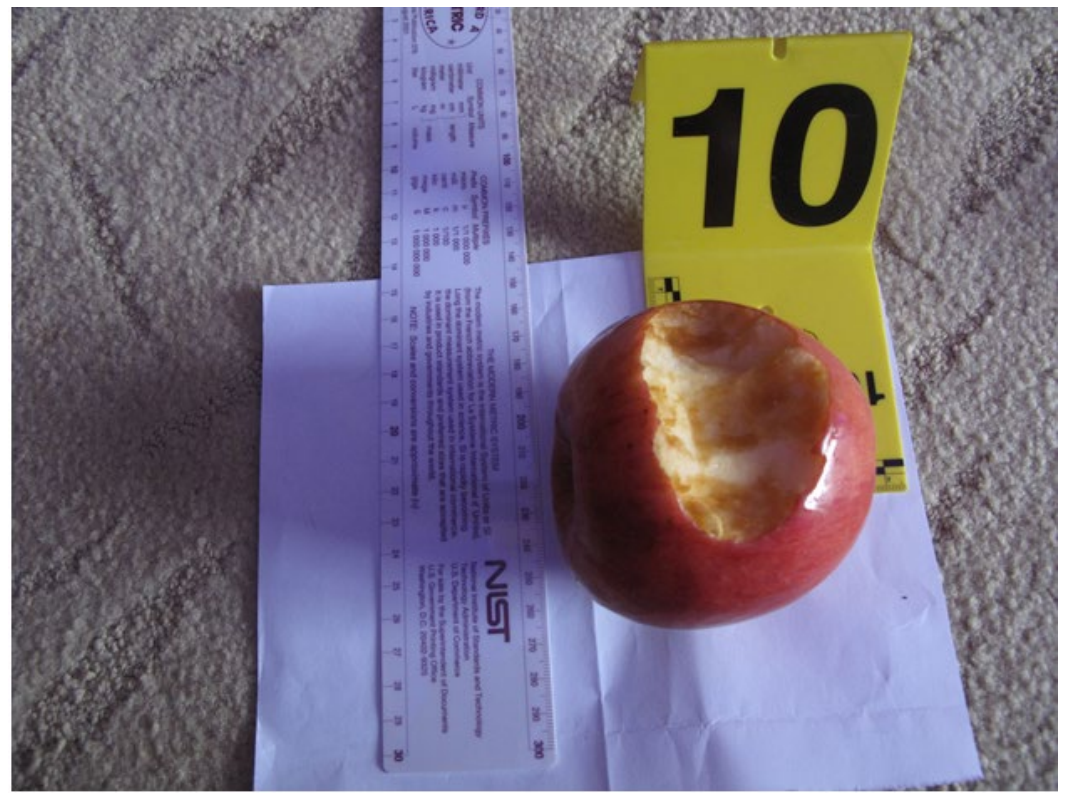

Figure 4: A bitten apple was photographed by a regular digital camera where no saliva stain was visible to the naked eye.

\section{Quasi-Experiment 3:}

The third volunteer was asked to spit onto a piece of bath towel several times. The bath towel was thrown onto the floor. The towel was laid there for two hours and then was photographed for a comparison purpose. The scenario was designed for a common situation where the suspect spits onto some types of fabric material (bedsheets, pillow-cases, or clothing) at a scene. To the naked eye, no latent saliva stain was visible at the time (See Figure 6).

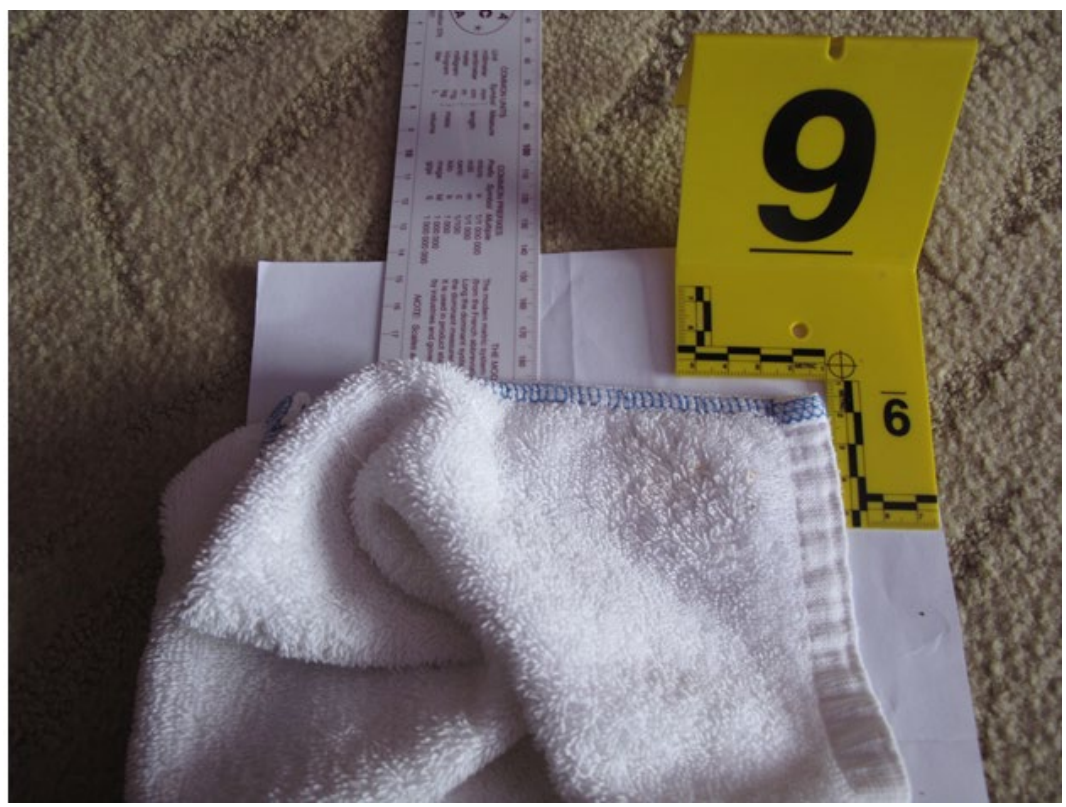

Figure 6: A piece of bath towel was photographed by a regular digital camera where no saliva stain is visible to the naked eye.

\section{Results:}

After two hours of waiting, the author began to photograph the three objects with a regular digital camera and no saliva stains could be seen on the surfaces (See Figure 2, 4, and 6). Then the author employed the laser hand-piece to scan each piece of the items with a yellow goggles on. Immediately, a larger area of dry saliva stain with a clear image was visible under the yellow goggle/filter. In other words, an excellent image of a large dry sa- 
liva stain was successfully detected, visualized, and photographed on the piece of kitchen paper towel using the laser device at 447 nm wavelength (See Figure 3). When the same procedure was applied to the bitten apple, a stripe-shape of noticeable dry saliva stain could be seen easily under the same laser beam (See Figure 5). Finally, when the laser hand-piece scanned the bath towel, a vague image of the spotted dry saliva stains became noticeable
(See Figure 7). Therefore, three levels of image quality of the latent dry saliva stains were detected, visualized and photographed from three difficult surfaces under the quasi-experimental research design with before-after comparison procedure. The diagram below summarizes the comparison results of the dry saliva stains on the three difficult surfaces in the study.

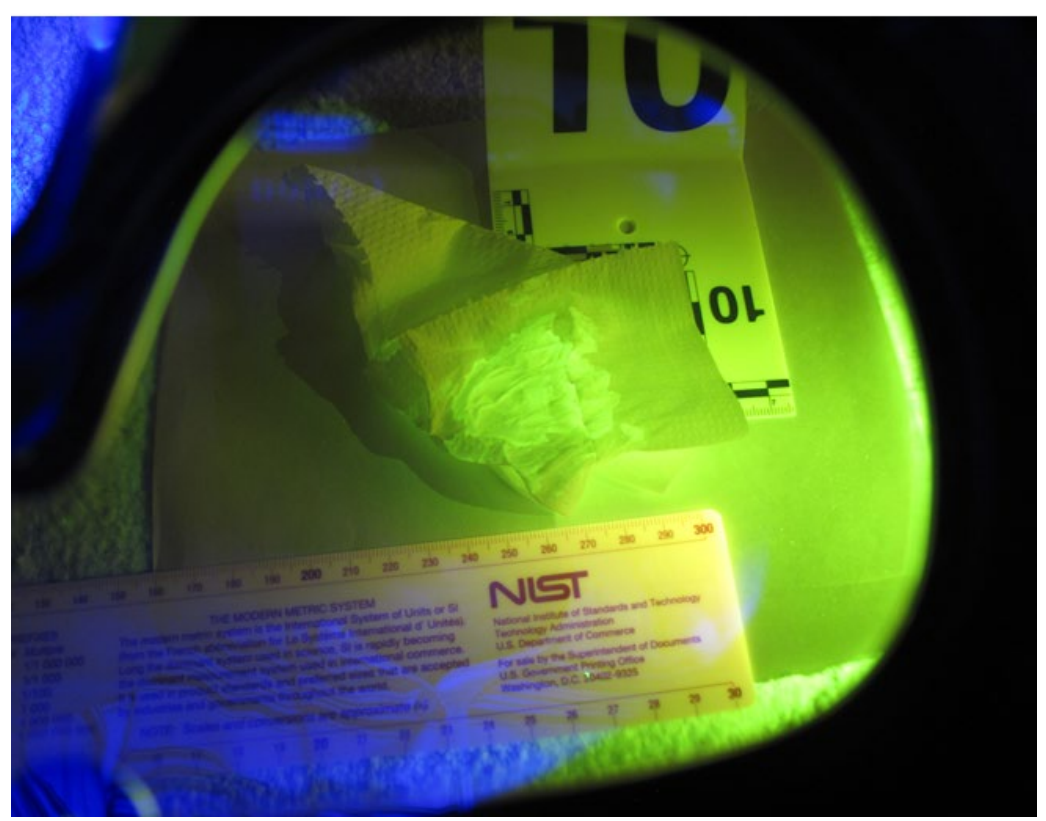

Figure 3: The same piece of the kitchen paper towel was photographed by the laser device under $447 \mathrm{~nm}$ and a yellow goggle. A fluorescent image (a high-level quality) of a large dry saliva stain was visible now.

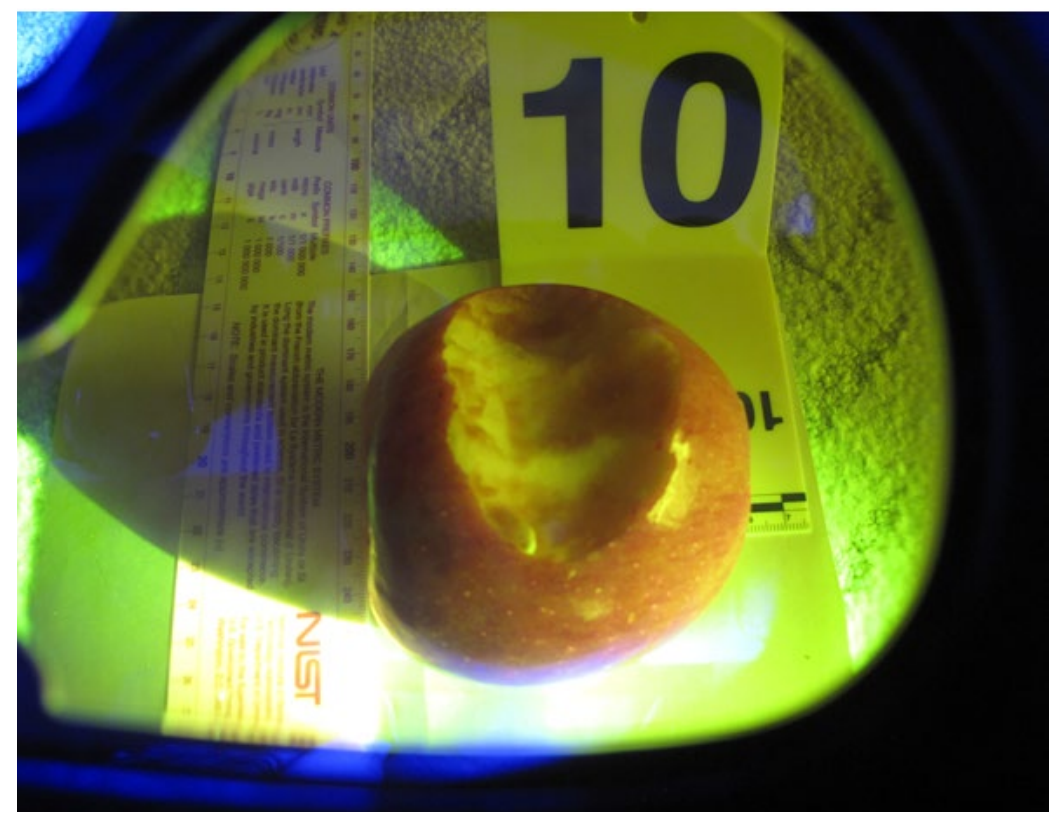

Figure 5: The same bitten apple was photographed by the laser device under $447 \mathrm{~nm}$ and a yellow goggle. A fluorescent image (a middle level quality) of a stripe-shape dry saliva stain was visible now. 


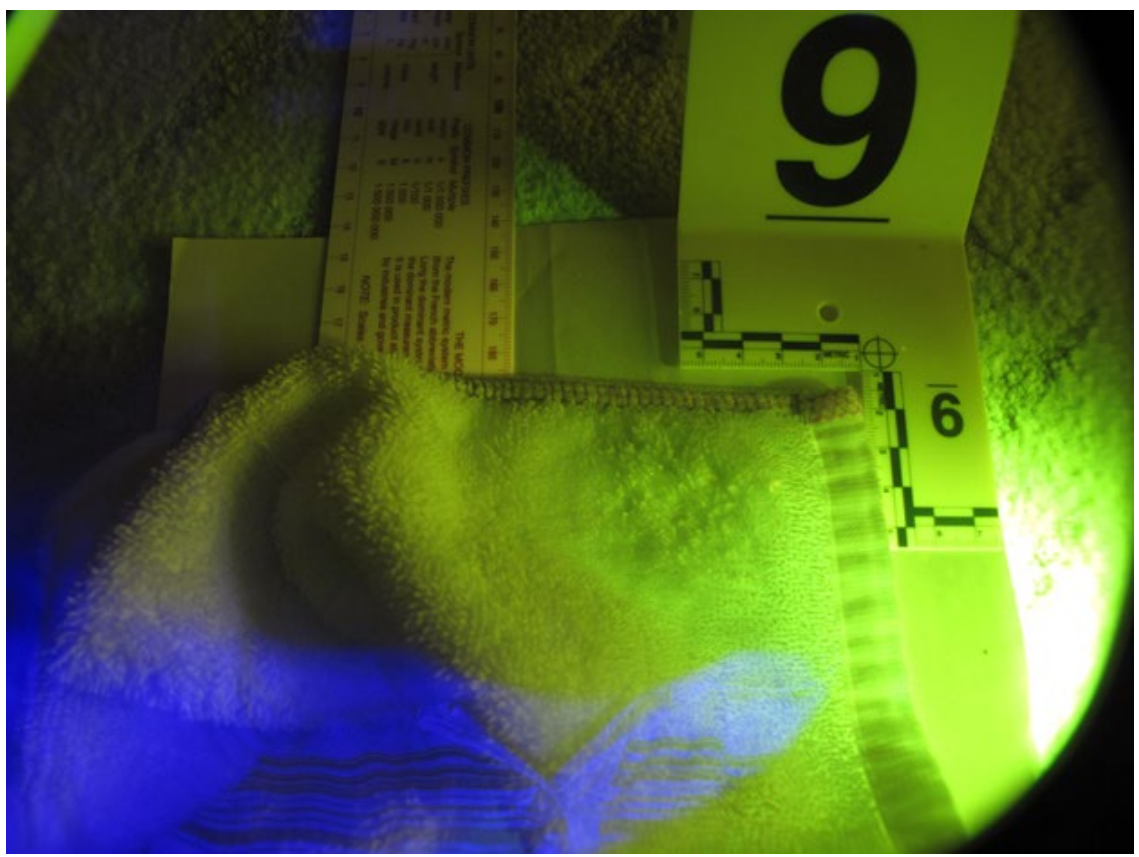

Figure 7: The same bath towel was photographed by the laser device under $447 \mathrm{~nm}$ and a yellow goggle. A fluorescent image (a low-level quality) of spotted dry saliva stains was visible now.

Table 1: Dry saliva stains were detected, visualized, and photographed using a blue laser $(447 \mathrm{~nm})$ and a yellow google/filter.

\begin{tabular}{|c|c|c|c|c|c|c|}
\hline $\begin{array}{c}\text { Types of } \\
\text { Object }\end{array}$ & $\begin{array}{c}\text { Types of } \\
\text { Surfaces }\end{array}$ & $\begin{array}{c}\text { Types of } \\
\text { Forces }\end{array}$ & Types of Acts & $\begin{array}{c}\text { Laser } \\
\text { Wavelength }\end{array}$ & $\begin{array}{c}\text { Goggle/Filter } \\
\text { Used }\end{array}$ & Image Results \\
\hline $\begin{array}{c}\text { Kitchen Paper } \\
\text { Towel }\end{array}$ & Paper & Heavy & Wiping & $447 \mathrm{~nm}$ & Yellow & Excellent \\
\hline Apple & Fruit & Free & Biting & $447 \mathrm{~nm}$ & Yellow & Clear \\
\hline Bathe Towel & Fabric & Natural & Spitting & $447 \mathrm{~nm}$ & Yellow & Noticeable \\
\hline
\end{tabular}

In sum, the laser beam was emitted at the $447 \mathrm{~nm}$ wavelength with the color purity level of $1 \mathrm{~nm}$ to minimize all light divergence. Subsequently, the presence of human latent dry saliva stains was detected, visualized, and photographed by observing the fluorescent images.

\section{Discussions:}

The scientific principle of the laser detection involves the photochemical fluorescent process. The photochemical rule, in a simple term, regulates a chemical reaction caused by absorption of light spectrum 100 400 nm as ultraviolet, $400 \sim 750 \mathrm{~nm}$ as visible, and $750 \sim 2500$ as infrared radiation. Due to the laser high energy encountered, a photochemical process produces non-destructive reactions much quicker than that via a thermal process. Photoexcitation is part of the photochemical process. According to the Grotthuss-Draper law, certain substance can be elevated or excited to a state of higher energy (excited state or fluorescence). In this study, the blue laser beam is almost monochromatic and has a relatively narrow band that can be efficiently used as the light source to provide the activation energy for the reaction from dry human saliva ${ }^{[10]}$. Therefore, it is possible to excite a molecule selectively and to produce a desired electronic and vibrational state, in this project, the re-emitted fluorescent images of the dry saliva stains under the yellow filter or goggles.

While lasers are unique optical lights due to their wavelength in a vacuum, most "single wavelength" lasers actually produce radiation in several modes with slightly different frequencies (wavelengths), which is often not in a single polarization. The temporal coherence mentioned earlier may mean mono-chromaticity; however, lasers emit a broad spectrum of light or emit different wavelengths of light simultaneously. In reality, lasers are not by nature single spatial mode and consequently produce light beams that diverge more than is required by the diffraction limit on different types of surfaces/substrates, thus explaining the three different levels of fluorescent images on the three different surfaces based their different absorption speeds. Therefore, a special improved technology is needed to further purify the laser beam. 


\section{Conclusion:}

The preliminary purpose of this project was to employ a new type of blue laser device $(447 \mathrm{~nm})$ and to detect and visualize latent dry saliva stains deposited on three difficult surfaces: a piece of kitchen paper towel, a bitten apple, and a piece of bath towel. The scenario in the quasi-experimental design consists of three levels of specific acts: wiping, biting, and spitting to imitate three types of possible acts by suspects at scenes. The preliminary results from the current study indicate that a much higher quality of a dry saliva stain was detected, visualized, and photographed from a piece of kitchen paper towel, a middle level of image was achieved from the bitten apple, and a lower level of image (still noticeable) was obtained from the bath towel.

Several advantages of this blue laser device can be summarized directly from the quasi-experimental project, compared with other similar products. First, the light weight $(3.5 \mathrm{~kg}$.) and the compact size $(225 \mathrm{~mm} / 9$ " (L.), $105 \mathrm{~mm} / 4.2$ "(W.), and $165 \mathrm{~mm} / 6.6$ "(H.)) qualifies for a portable usage for a field as well as a lab applications. The battery $(1.5 \mathrm{~kg})$ to run the operation is detachable and insertable and ready to use immediately without any starting time for approximately $4 \sim 6$ hours. Second, the laser device has a dual wavelength (447 nm or $532 \mathrm{~nm}$ ) with a switch for selection, which allows wider applications (to be reported in coming research studies). Third, using a special TE automatic cooling control system, the delivery system (the hand-piece) with a continuous adjustable focus can minimize the interference of the laser beam and produce a spot area ( $D=50$ centimeters) without any speckles in the image, for which similar laser products suffer. Next, while an UV light must require a dark setting to see an image, this laser device can generate a very strong blue laser beam and excite human dry saliva stains to be fluorescent for visualization and photographing under a normal daylighting. Finally, the photographing is also easy to operate with a regular digital camera and a regular yellow filter. In fact, the goggle provided can be used as a filter as well because both can effectively block the laser light source, isolate the excited fluorescence, and in some cases reduce the exhibited fluorescence from the surfaces. In some cases, certain substrates also exhibit fluorescence, which can partially or totally obscures the re-mitted image. Therefore, the combination of the new brand laser device $(447 \mathrm{~nm})$, the special hand-piece, and the yellow goggle/filter can add significantly to both the extent and clarity of fluorescing images of the laser-treated dry saliva stains that would otherwise remain undetected using other traditional development methods.

All of these observations from the quasi-experimental project should render the laser device a practical instrument for crime scene processing and identification in the field as well as in the lab. In sum, such a device may reduce the time required to process a crime scene by providing rapid and real-time detection, visualization, and photographing to forensic investigators.

While the image quality of the latent dry saliva stains in this study tend to be a promising future for forensic examination at the scene or in the lab, many new questions remain for further development. First, more quasi-experimental studies are needed for latent dry saliva detection on other difficult surface, such as leather (belt and shoes), polyester fabric (bras and underwear), and human skin (kissing areas and bite-marks). Second, the quasi-experimental study is limited to detect dry saliva deposition with two hours only, future studies should be extended for longer deposition hours. Third, to explore the utility of the laser technique, future locations should be moved from the indoor setting in this study to outdoor settings. Next, while the laser method works on latent dry saliva stains, it may be also applicable on other latent biological evidence, such as semen, blood, and/or virginal fluid. Finally, a similar project may be workable on latent sweaty fingerprints on some difficult surfaces. In conclusion, it is believed that this portable laser device, after a few years of studying and quasi-experimenting, should have wider applications for forensic examination in the field and in the lab due to its non-destruction (DNA free), non-contact, and non-invasive nature.

\section{Acknowledgement:}

The author would gratefully acknowledge technical supports for this research project from Suzhou Science \& Technology Development Co., Ltd., China where the device is manufactured and provided.

\section{References:}

1. Sweet D.; Lorente, M.; Valenzuela A.; Lorente J.A.; Alvarez, J.C.; Increasing DNA Extraction Yield from Saliva Stains with a Modified Chelex Method. Forensic Sci Int. 1996, 83:167-77.

2. Kirk, P.L. Crime Investigations. New York: Interscience Publishers Inc., 1953, p 103.

3. Sweet, D.; Lorente, M.; Lorente, J.A.; Valenzuela, A.; Villanueva, E. An Improved Method to Recover Saliva from Human Skin: The Double Swab Technique. J. Forensic Sci. 1997, 84:168-174.

4. Walsh, D.J.; Corey, A.C.; Cotton, R.W.; Forman, L.; Herrin, G.L.; Word, C.J. et al. Isolation of DNA from saliva and forensic science samples containing saliva. J Forensic Sci. 1992, 37: 387-395.

5. Virkler, K. Analysis of Body Fluids for Forensic Purposes: From Laboratory Testing to Non-Destructive Rapid Confirmatory Identification at a Crime Scene. Forensic Sci Int. 2009, 188: (1-3): $1-17$.

6. Kanwar, D. N.; K Ranganathan; Umadevi, K.M.; Joshua, E. A Rapid and Noninvasive Method to Detect Dried Saliva Stains from Human Skin Using Fluorescent Spectroscopy. J. Oral Maxillofac Pathol. 2011, 15(1): 22-25.

7. Auvdel, M.J. Comparison of Laser and Ultraviolet Techniques Used in the Detection of Body Secretions. Journal of Forensic Sciences, 1987, 32 (2): 326-345.

8. Wang, Z. J. Quantitative Comparison of Partial-Full Fingerprints at Scenes Using a Hand-Held Digital Device: A Quasi-Experimental Design Study. J. Forensic Investigation, 2016, 4 (2): 1-6.

9. Price, P. Psychology Research Methods: Core Skills and Concepts. Flat World Knowledge Publisher: Irvington, NY, 2012.

10. Glusac, K. What Has Light Ever Done for Chemistry? Nature Chemistry 2016, 8 (3): 734- 773. 